Technological University Dublin

DÜBLIN

ARROW@TU Dublin

Articles

School of Mathematics

1988-01-01

\title{
Essentially Indecomposable Modules Which Are Almost Free
}

Brendan Goldsmith

Technological University Dublin, brendan.goldsmith@tudublin.ie

R. Gobel

Follow this and additional works at: https://arrow.tudublin.ie/scschmatart

Part of the Mathematics Commons

\section{Recommended Citation}

Goldsmith, B. \& Gobel:, R. (1988). Essentially indecomposable modules which are almost free. Quarterly Journal of Mathematics Oxford, vol. 2, no. 39, pg. 213-222. doi:10.1093/qmath/39.2.213

This Article is brought to you for free and open access by the School of Mathematics at ARROW@TU Dublin. It has been accepted for inclusion in Articles by an authorized administrator of ARROW@TU Dublin. For more information, please contact arrow.admin@tudublin.ie, aisling.coyne@tudublin.ie,gerard.connolly@tudublin.ie.

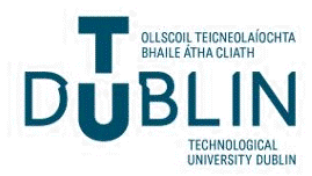




\title{
Essentially Indecomposable Modules Over
} a Complete Discrete Valuation Ring.

\author{
B. GoLdsMith $(*)$
}

\section{Introduction.}

Torsion-free modules over a complete discrete valuation ring $R$ are markedly different from abelian groups or modules over an incomplete discrete valuation ring in that the only indecomposable modules which exist have rank 1 and so are isomorphic to $R$ itself or the field, $Q$, of fractions of $R([7], p .45)$. In this paper we investigate how close a reduced torsion-free $R$-module of infinite rank can come to being indecomposable. In particular we establish in $\$ 4$ the existence of essentially indecomposable modules with basic submodules of countable rank. The results here bear a strong resemblance to results on $p$-groups $([2],[5],[9]$ and $[10])$. Notation follows the standard works of Fuchs [3], [4] while set-theoretic concepts, which are kept to a minimum, may be found in Jech [6].

\section{Maximal pure submodules.}

Let $R$ denote a complete discrete valuation ring of cardinality $\nu$ with unique prime $p$. For an infinite cardinal $\lambda$ we let $S_{\lambda}$ (or just $S$ if no ambiguity is possible) denote a free $R$-module of rank $\lambda$. Clearly $S_{\lambda}$ is not complete in the $p$-adic topology and we denote its completion by $\hat{S}_{\lambda}$ (or just $\hat{S}$ ).

(*) Indirizzo dell'A.: Dublin Institute of Technology, Dublin 8, Treland and Dublin Institute for Advanced Studies, Dublin 4, Ireland. 
DEFINITION. A $R$-module $X$ is said to be a maximal pure submodule of the complete $R$-module $S$ if $X$ is a pure submodule of $S$ containing $S$ and $S / X \cong Q$, the field of fractions of $R$. We remark that if $X$ is a maximal pure submodule of $\hat{S}$ then for any $x \in \hat{S} \backslash X$, we have $\hat{S}=\langle X, x\rangle_{*}$.

LEmma 2.1. If $G$ and $K$ are pure submodules of $S$ containing $S$ then $G \cong K$ if and only if there is an automorphism $\theta$ of $\hat{S}$ with $G \theta=K$.

Proof. The sufficiency is clear, we establish necessity. Let $\varphi$ be an isomorphism from $G$ onto $K$. Then $\varphi$ extends uniquely to an endomorphism $\hat{\varphi}$ of $\widehat{S}$. Similarly if $\psi$ is the inverse of $\varphi$, it extends to an endomorphism $\hat{\psi}$ of $\hat{S}$. However since $G_{r}^{r}$ and $K$ are dense subsets of the Hausdorff space $S$, it follows easily that $\hat{\varphi} \hat{\psi}$ and $\hat{\varphi} \hat{\varphi}$ act as the identity on $\hat{S}$. Thus $\theta=\hat{\phi}$ is the required automorphism.

Before examining the endomorphism rings of maximal pure sub modules of $\hat{S}$, we introduce the concept of an inessential endomorphism (cf. [5]). Let $X$ be a pure submodule of $\hat{S}$ containing $S$, then, as we noted in the proof of Lemma 2.1, any endomorphism $\varphi$ of $X$ has a unique extension $\hat{\varphi}$ to an endomorphism of $\hat{S}$. We define an endomorphism of $X$ to be inessential if its mique extension to $S$ maps $S$ into $X$. It is easily seen that the difference of two inessential endomorphisms is inessential while the composition of two endomorphism is inessential when either factor is inessential. Thus the inessential endomorphisms of $X$ form a two-sided ideal $I(X)$ in the endomorphism ring $E(X)$ of $X$.

THeorem 2.2. For any infinite cardinal $\lambda$, there exists an $R$-module $G$, with basic submodule of rank $\lambda$, such that $E(G)$ is the ring split extension of $R$ by $I(G)$,

$$
E(G)=R \oplus I(G)
$$

Proof. Let $\$$ be a free $R$-module of rank $\lambda$ and let $G$ be any maximal pure submodule of $S$. Clearly $S$ is basic in $G$. We may identify $E(G)$ as a subring of $E(S)$ by identifying each endomorphism in $E(G)$ with its unique extension $\hat{\varphi}$ in $E(\hat{S})$. With this identification $I(G)$ is a left ideal of $E(\hat{S})$.

Pick $x \in \hat{S} \backslash G$. Then for arbitrary $\varphi$ in $E(G)$ we must have $q(x \hat{\varphi})=$ $=t x+g$, some $q, t \in R, g \in G$. Since every element of $R$ is a product of a power of $p$ and a unit, there is no loss in generality in supposing 
$q=p^{r}, t=p^{s}$. We consider two cases:

(i)

$r \leqslant s$.

In this case $p^{r}\left(x \hat{\varphi}-p^{s-r} x\right)=g$. The purity of $G$ in $S$ implies that $x\left(\hat{\varphi}-p^{s-r} 1\right)$ belongs to $G$. Since $G$ is invariant under $\hat{\varphi}-p^{s-r} 1$ and $\langle G, x\rangle_{*}=\hat{S}$, it is clear that $\hat{S}\left(\hat{\varphi}-p^{s-r} 1\right)$ is contained in $G$. Thus $\hat{\varphi}-p^{s-r} 1 \in I(G)$ and so $E(G)=R+I(G)$.

(ii)

$$
r>s \text {. }
$$

We show that this case cannot arise. As before we can show that $x\left(p^{r-s} \hat{\varphi}-1\right) \in G$ and deduce that $p^{r-s} \hat{\varphi}-1 \in I(G)$. Suppose $p^{r-s} \hat{\varphi}-$ $-1=\theta$, where $\theta \in I(G)$. Since $r>s, p^{r-s} \hat{\varphi}$ belongs to the Jacobson. radical of $E(\hat{S})$ (see [8]) and this forces $\theta$ to be a unit in $E(\widehat{S})$. However since $G$ is certainly not a homomorphic image of $\hat{S}, I(G)$ is a proper left ideal of $E(\hat{S})$ which contains a unit-contradiction. So case (ii) does not arise.

Since $G$ is pure in $\hat{S}, R \cap I(G)=0$ and $q u \hat{a}$ modules, $E(G)=R \oplus$ $\oplus I(G)$. However this is cleariy a ring split extension also and we have established the result.

\section{Essentially-rigid systems of $R$-modules.}

As we noted in the introduction indecomposable $R$-modules have rank 1 whereas indecomposable abelian groups of arbitrary large rank exist [11]. One useful tool in the investigation of indecomposable abelian groups was the concept of a rigid system of groups (see [4] $\S 88)$. In this section we define and explore an analagous concept for $R$-modules.

We extend the concept of inessential to homomorphisms between different reduced torsion-free $R$-modules $X_{i}, X_{j}$ by defining $I_{i}\left(X_{j}\right)=$ $=\left\{\varphi \in \operatorname{Hom}\left(X_{i}, X_{j}\right) \mid \hat{X}_{i} \hat{\varphi} \leqslant X_{i}\right\}$ where $\hat{\varphi}$ denotes the unique extension of $\varphi$ to a map $\hat{X}_{i} \rightarrow \hat{X}_{i}$.

Defrnttron. A family $\left\{X_{j}\right\}(j \in J)$ of reduced torsion-free $R$-modules is said to be essentially rigid if

$$
\operatorname{Hom}\left(X_{i}, X_{j}\right)= \begin{cases}R \oplus I\left(X_{j}\right) & \text { if } i=j \\ I_{i}\left(X_{j}\right) & \text { if } i \neq j,\end{cases}
$$

for all $i, j \in J$. 
Suppose throughout this section that $R$ is a complete discrete valuation ring of cardinality $\nu$ and $\lambda$ is an infinite cardinal satisfying $\mu=\lambda^{N_{0}}=2^{\lambda}$ and $v \leqslant \mu$. For an infinite cardinal $\sigma$, let $\sigma^{+}$denote the successor of $\sigma$. We can now state the main result of this section.

THEOREM 3.1. If $\lambda$ is an infinite cardinal with the property that $\mu=\lambda N_{0}=2^{\lambda}$ and $R$ is a complete discrete valuation ring of cardinality $\nu \leqslant \mu$, then there exists an essentially-rigid family of $R$-modules having $\mu^{+}$members.

Remark. (i) By assuming G.C.H. we may, of course replace $\mu^{+}$ by $2^{\mu}$.

(ii) Cardinals of the form $\lambda$ do exist for values of $\lambda$ other than $\lambda=\boldsymbol{\aleph}_{0}$ e.g. assuming G.C.H., any cardinal of cofinality
$\aleph_{0}$ will do.

LiEMma 3.2. Let $V$ be a vector space of dimension $\alpha$, an infinite cardinal, over a field. Let $\left\{W_{i}\right\}(i<\beta)$ be a family of subspaces of $V$ indexed by the cardinal $\beta \leqslant \alpha$, such that $\operatorname{dim} W_{i}=\alpha$ for all $i<\beta$. Then there exist $\alpha^{+}$subspaces $\{U\}$ of $V$ such that each subspace $U$ is of codimension 1 in $V$ and no subspace $W_{i}$ is contained in any subspace $U$.

Proof. By a result of Beaumont and Pierce ([1]), Lemma 5.2) there exists at least one such subspace, $U_{0}$ say. Suppose that the subspaces $\left\{V_{i}\right\} \quad(i<\zeta)$ have been constructed and $\zeta<\alpha^{+}$. Then the set of subspaces consisting of the given $W_{i}$ together with the constructed subspaces $U_{i}$ constitutes a family of at most $\alpha$ subspaces each of dimension $\alpha$. Applying Beaumont and Pierce's result to this family yields another subspace of codimension 1. Call this subspace $U_{\zeta}$. The result follows easily by tramsfinite induction.

LemaA 3.3. If $S$ is free of rank $\lambda$ then there exist $\mu^{+}$maximal pure submodules of $\hat{S}$ with the property that none of them contains a submodule isomorphic to $S$.

PRoof. Since $S$ is free of $\operatorname{rank} \lambda,|\hat{S}|=\max \left(\lambda \kappa_{0}, \nu_{0}\right)$. But $\nu \leqslant \mu$ implies that $\nu^{\boldsymbol{N}_{0}} \leqslant \mu^{\boldsymbol{N}_{0}}=\left(\lambda^{\boldsymbol{N}_{0}}\right)^{\boldsymbol{N}_{0}}=\lambda \boldsymbol{N}_{0}=\mu$. So $\hat{S} / S$ is a Q-vector space of dimension $\mu=\lambda \boldsymbol{N}^{\boldsymbol{N}_{0}}$. Now let $\left\{W_{k}\right\}(k \in K)$ be the collection of submodules of $\hat{S}$ which are isomorphic to $\hat{S}$. Fach of these submodules is determined by an endomorphism of $\hat{S}$. However each endomorphism 
of $S$ is completely determined by its action on $S$ which has rank $\lambda$, so $|E(\hat{S})|=\mu^{\lambda}=\left(2^{\lambda}\right)^{\lambda}=2^{\lambda}=\mu$. Hence $\left\{W_{k}\right\}(k \in K)$ is a family of at most $\mu$ submodules of $\hat{S}$.

Let $\bar{W}_{k}=\left\langle W_{k}+S\right\rangle_{*} / S$. Then $\left\{\bar{W}_{k}\right\} \quad(k \in K)$ is a family of at most $\mu$ subspaces of the $Q$-vector space $S / S$ which has dimension $\mu$. Since $\bar{W}_{k} \cong\left(Q \otimes_{R}\left(W_{k}+S\right)\right) / Q \otimes_{R} S$, it follows that each $\bar{W}_{k}$ has dimension $\mu$. By Lemma 3.2 we can find $\mu^{+}$subspaces $U$ such that no $\bar{W}_{k}$ is contained in any $U$ and, moreover, each $U$ has codimension 1 in $S / S$. If $G$ is a submodule of $S$ with $G / S=U$, then $G$ is a maximal pure submodule of $\hat{S}$ and clearly no $W_{k}$ is contained in any $G$. Thus we have constructed the required family of $\mu^{+}$maximal pure submodules.

Let $G_{2}$ denote the collection of all maximal pure submodules of $\widehat{S}_{\lambda}$ which do not contain an isomorphic copy of $\vec{S}_{\lambda}$.

Lemra 3.4. If $\left\{G_{\alpha}\right\}(\alpha<\beta)$ is a subset of $\mathcal{G}_{2}$ and $|\beta| \leqslant \mu$, then there exist $\mu^{+}$submodules $G$ in $\mathcal{G}_{\lambda}$ such that $\operatorname{Hom}\left(G_{\alpha}, G\right)=I_{\alpha}(G)$ for all $\alpha<\beta$.

Proof. The proof is similar to that of Lemma 3.3. Suppose $\left\{W_{\alpha_{i}}\right\}$ denotes the set of endomorphic images of $G_{\alpha}$ which have rank $\mu$. Then for each $\alpha$, the set $\left\{W_{\alpha_{i}}\right\}$ is of cardinality at most $\mu$. Since $|\beta| \leqslant \mu$, the union of all such collections is a set of at most $\mu$ submodules of $\hat{S}$. Call this set $w$. Now let $\vartheta$ denote the set of endomorphic images of $\hat{S}$ which are isomorphic to $\hat{S}$. Then $w \cup \mathcal{V}$ is a collection of at most $\mu$ submodules of $\widehat{S}$, say $\mathcal{W} \cup \mathcal{V}=\left\{X_{i}\right\}(i<\mu)$. Note that each $X_{i}$ has rank $\mu$. Set $\bar{X}_{i}=\left\langle X_{i}+S\right\rangle_{*} / S$; then $\left\{\bar{X}_{i}\right\}(i<\mu)$ is a collec tion of $\mu$ subspaces of the $Q$-vector space $S / S$ and each $\bar{X}_{i}$ has dimension $\mu$. By Lemma 3.2 there exist $\mu^{+}$maximal subspaces $U$ such that no $\bar{X}_{i}$ is contained in a $U$. Choose a maximal pure submodule $G$ such that $G / S=U$. Clearly $G \in \mathcal{G}_{\lambda}$.

Now consider Hom $\left(G_{\alpha}, G\right)$ for any $\alpha$. If $\varphi: G_{\alpha} \rightarrow G$ is not inessential then Ker $\hat{\varphi}$ is contained in $G_{\alpha}$ which forces Ker $\hat{\varphi}$ to have rank less than $\mu$. But then $\operatorname{Im} \varphi \cong G_{\alpha} / \operatorname{Ker} \varphi$ is an endomorphic image of $G_{\alpha}$ of rank $\mu$ and is contained in $G$-contradiction. So we conclude that $\operatorname{Hom}\left(G_{\alpha}, G\right)=I_{\alpha}(G)$ for each $\alpha$.

Lemrora 3.5. Given any maximal pure submodule $G$ of $\vec{S}_{\lambda}$, there are at most $\mu$ maximal pure submodules $G_{i}$ of $\hat{S}_{\lambda}$ for which $\operatorname{Hom}\left(G_{i}\right.$, $G) \neq I_{i}(G)$. 
Proof. Suppose there exists a family $\left\{G_{i}\right\}(i \in J)$ of more than $\mu$ submodules. For each $i \in J$, pick a homomorphism $\varphi_{i}: G_{i} \rightarrow G$. Then $\left\{\hat{\varphi}_{i}\right\}(i \in J)$ is a family of more than $\mu$ endomorphisms of $\hat{S}_{\lambda}$. Since $\left|E\left(\hat{S}_{\lambda}\right)\right|=\mu$, we must have $\hat{\varphi}_{i}=\hat{\varphi}_{j}$ for some $i \neq j \in J$. But then

$$
\hat{S} \hat{\varphi}_{i}=\left(G_{i}+G_{j}\right) \hat{\varphi}_{i} \leqslant G_{i} \hat{\varphi}_{i}+G_{j} \hat{\varphi}_{j}=G .
$$

Thus $\varphi_{i}$ is inessential-contradiction. This ostablishes the lemma.

Proof of Theorein 3.1. Choose $G_{0}$ to be any member of $\mathcal{G}_{\lambda}$. Suppose the essentially-rigid family $\left\{G_{\alpha}\right\}(\alpha<\beta)$ has been constructed for $\beta<\mu^{+}$. By Lemma 3.4 there exist $\mu^{+}$maximal pure submodules $G$ such that $\operatorname{Hom}\left(G_{\alpha}, G\right)=I_{\alpha}(G)$. However for each $\alpha<\beta$, there are, by Lemma 3.5, at most $\mu$ of these submodules $G$ for which Hom ( $G$, $\left.G_{\alpha}\right) ; I\left(G_{\alpha}\right)$. Then deleting all such submodules $G$ deletes at most $\mu$ submodules from the original collection since $\beta \leqslant \mu$. So there exists $G \in \mathcal{G}_{2}$ with $\operatorname{Hom}\left(G, G_{\alpha}\right)=I\left(G_{\alpha}\right)$ and Hom $\left(G_{\alpha}, G\right)=I_{\alpha}(G)$ for all $\alpha<\beta$. Set $G_{\beta}=G$. Then the family $\left\{G_{\alpha}\right\} \quad(\alpha \leqslant \beta)$ is essentially-rigid. The proof is completed by transfinite induction.

\section{Essentially-indecomposable modules.}

In this section we show that a slightly stronger result than Theorem 3.1 can be deduced and apply this new result to the construction of essentially indecomposable modules.

We shall use the term basic rank of a homomorphism to denote the rank of a basic submodule of the image of the homomorphism.

DeFinition. If $S$ is a free $R$-module of infinite rank $\lambda$ and $X$ is a pure submodule of $S$ containing $S$, then we define

$$
I_{\lambda}(X)=\{\varphi \in E(X) \mid \hat{S} \hat{\varphi} \leqslant X \text { and } \hat{\varphi} \text { has basio } \operatorname{rank}<\lambda\}
$$

Clearly $I_{\lambda}(X)$ is an idesul in $E(X)$.

THEOREM 4.1. If $R$ is a complete discrete valuation ring of cardinality $\gamma$ and $\lambda$ is an infinite cardinal such that $\mu=\lambda^{N_{0}}=2^{\lambda}$ and $y \leqslant \mu$, then there exists a family of $\mu^{+} R$-modules $\left\{G_{j}\right\}(j \in J)$ such that 
more than $\mu$ $\rightarrow$ G. Then of $\hat{S}_{\lambda}$. Since but then

lemma.

r of $\boldsymbol{G}_{\lambda}$. Supconstructed submodules $G$ $\beta$, there are, which $\operatorname{Hom}(G$, etes at most $\mu$ So there exists G) for all $\alpha<\beta$. lly-rigid. The

nit than Thethe construeisma to denote omomorphism. ank $\lambda$ and $X$ $<\lambda\}$

In ring of car$\lambda x_{0}=2^{\lambda}$ and $(j \in J)$ such (i) for each $j \in J, E\left(G_{j}\right)=R \oplus I_{\lambda}\left(G_{j}\right)$;

(ii) for distinct $j, k \in J$, every homomorphism $G_{j} \rightarrow G_{k}$ is inessential and has basic rank less than $\lambda$

Proof. This stronge result comes by observing in the proof of Theorem 3.1 that all of the modules constructed actually belong to $\mathcal{G}_{\lambda}$. Since the image of an inessential homomorphism is complete, it must be the completion of a free module of rank less than $\lambda$. This gives the desired result.

Recall that $E_{0}(G)$ denotes the ideal of $E(G)$ consisting of all endomorphisms of finite rank.

CoRollary 4.2 (G.C.H.). If $R$ is a complete discrete valuation ring of cardinality $2^{\mathrm{N}_{0}}$, then there exists a family of $2^{2_{0}} R$ modules $\left\{G_{j}\right\}(j \in J)$ each with basic submodules of rank $\aleph_{0}$ such that

(i) for each $j \in J, E\left(G_{j}\right)=R \oplus E_{0}\left(G_{j}\right)$;

(ii) for distinet $j, k \in J$, every homomorphism $G_{j} \rightarrow G_{z}$ has finite rank.

Proof. Since $2^{\boldsymbol{N}_{0}} \leqslant\left(\boldsymbol{N}_{0}\right)^{\boldsymbol{N}_{0}} \leqslant\left(2^{\boldsymbol{N}_{0}}\right)^{\boldsymbol{N}_{0}}=2^{\boldsymbol{*}_{0}}$, we see that $\lambda=\boldsymbol{\aleph}_{0}$ satisfies the cardinality requirements of Theorem 4.1. However if a homomorphism from $G_{j}$ has finite basic rank then it clearly also has finite rank. The result now follows from Theorem 4.1 and G.O.H.

DEFINITION. If $\lambda$ is an infinite cardinal we say that a reduced torsion-free $R$-module $G$ is $\lambda$-essentially indecomposable if in any decomposition $G=A \oplus B$, one of $A, B$ is the completion of a free module of rank less then $\lambda$.

In the case $\lambda=s_{0}$ we are requiring that in any direct decomposition one of the summands is complete of finite rank. A module with this property is said to be essentially indecomposable (cf. essentially indecomposable $p$-groups, $[9], \S 15$ ).

The existence of $\lambda$-essentially indecomposable modules follows rather easily from Theorem 4.1 in the case $\lambda^{\kappa_{0}}=2^{\lambda}$. For if $G$ is one of the modules constructed in Theorem 4.1 and we have a decomposition $G=A \oplus B$ with associated projections $\pi_{1}$ and $\pi_{2}$, then one of $\pi_{1}, \pi_{2}$ belongs to $I_{\lambda}(G)$ since the quotient $E(G) / I_{\lambda}(G)$ is a domain. If $\pi_{1} \in$ $\in I_{\lambda}(G)$ then clearly $A$ is the completion of a free $R$-module of rank less then $\lambda$. In particular if $\lambda=\aleph_{0}$ we have established the existence of $2^{{ }^{N_{0}}}$ essentially indecomposable $R$-modules for any complete discrete valuation ring $R$ of cardinality $2^{\kappa_{0}}$. 
We conclude this section by constructing an essentially indecomposable module which is not a maximal pure submodule of a complete module.

Propostmrow 4.3. If $R$ is a complete discrete valuation ring of cardinality $2^{*}$ and $S$ is a free $R$-module of countably infinite rank, then there exists a pure submodule $H$ of $\hat{S}$ containing $S$ with $\hat{S} / H \cong$ $\cong Q \oplus Q$ and such that $E(H)=R \oplus E_{0}(H)$

Proof. Choose distinct maximal pure submodules $G$ and $G$ belonging to the family construeted in Corollary 4.2. Set $H=G \cap G_{1}$. Clearly $S \leqslant H \leqslant \hat{S}$ and both inclusions are pure. Also $S / H \cong Q \oplus Q$. Let $S=\langle H, x, y\rangle_{*}$ where $G=\langle H, x\rangle_{*}$ and $G_{1}=\langle H, y\rangle_{*}$. Let $\varphi$ be any endomorphism of $H$. Then as in the proof of Theorem 2.2 we may write

$$
\begin{aligned}
& q(x \hat{\varphi})=h_{0}+\alpha x+\beta y \\
& q(y \hat{\varphi})=h_{1}+\gamma x+\delta y
\end{aligned}
$$

where $q, \alpha, \beta, \gamma, \delta \in R$ and $h_{0}, h_{1} \in H$.

Now $x(q \hat{\varphi}-\alpha 1) \in G_{1}$ and so $q \hat{\varphi}-\alpha 1$ maps $G$ into $G_{1}$. From the properties of $G$ and $G$ we conclude that $q \hat{\varphi}-\alpha 1$ is an inessential endomorphism. It follows as in the proof of Theorem 2.2, that $\hat{\varphi} \mid G$ belongs to $R \oplus I\left(G_{1}\right)$. Hence we can write $\phi=r+\theta$ where $r \in R$, $\theta \in I\left(G_{1}\right)$. But then $\theta \in E(H) \cap I\left(G_{1}\right)=E(H) \cap E_{0}(G)=E_{0}(H)$. So $\varphi \in R \oplus E_{0}(H)$ and $E(H) \leqslant R \oplus E_{0}(H)$. The reverse inequality is clearly true so $E(H)=R \oplus E_{0}(H)$.

Aotnowledgment. This paper is based on work in the anthor's D. Phil. thesis written under the supervision of Dr. A. L. S. Corner; I would like to express my appreciation of his encouragement and help.

\section{REFERENCES}

[1] R. A. Beaumont - R. S. Prerce, Some invariants of p-groups, Michigan Math. J., II (1964), pp. 137-149.

[2] A. L. S. CORNer, On endomorphism rings of primary abetian groups, Quart. J. Math. (Oxford) (2), 20 (1969), pp. 277-296. 
ing indecomif a complete

tion ring of finite rank, with $\hat{S} / H \cong$

a $G_{1}$ belong$\mathrm{H}=G \cap G_{1}$. $8 H \cong Q \oplus Q$.

Let $\varphi$ be grem 2.2 we

From the inessential 2, that $\hat{\varphi} \mid G$ where $r \in R$, $E_{0}(H)$. So lity is clearly

the author's L. S. Corner; grement and ups, Michigan belian groups,
[3] L. Fuchs, Infinite Abelian Groups - Vol. I, Academic Press, New York and London, 1970.

[4] L. Fuchs, Infinite Abelian Groups - Vol. II, Academic Press, New York and London, 1973.

[5] B. Goldsmith, Essentially-rigid families of abelian p-groups, J. London Math. Soc., (2), 18 (1978), pp. 70-74.

[6] T. J. JEcH, Lectures in Set Theory, Lecture Notes in Mathematics - Vol. 217, Springer-Verlag, Berlin 1971.

[7] I. Kaplansky, Infinite Abelian Groups, University of Michigan Press, Ann Arbor, 1954.

[8] W. LTEBERT, Characterisation of the endomorphism rings of divisible tor. sion modules and reduced complete torsion-free modules over complete discrete valuation rings, Pacific J. Math., $\mathbf{3 7}$ (1971), pp. 141-170.

[9] R. S. PIERCE, Homomorphisms of primary abelian groups, Topics in Abelian Groups, pp. 215-310, Scott, Foresman \& Co., Chicago, 1963.

[10] S. SHelaH, Existence of rigid-like families of abelian p-groups, Model Theory and Algebra, Lecture Notes in Mathematies Vol. 498, pp. 384402, Springer-Verlag, Berlin, 1975.

[11] S. SHELAH, Infinite Abelian Groups, Whitehead problem and some constructions, Israel J. Math., 18 (1974), pp. 243-256.

Manoscritto pervenuto in redazione il 13 Marzo 1982. 